\title{
Publisher Correction: Surface-wetting characterization using contact-angle measurements
}

Tommi Huhtamäki, Xuelin Tian (D), Juuso T. Korhonen and Robin H. A. Ras (D)

Correction to: Nature Protocols https://doi.org/10.1038/s41596-018-0003-z, published online 9 July 2018

The version of this Protocol originally published contained typographical errors that affected the accuracy/readability of the text. In Fig. 4e, the line "Contact angle remainsstable" should have read "Contact angle remains stable." In Table 1, in the "Advantages" column, the second instance of "Simple" was incorrectly associated with the "Sessile-drop goniometry" method; it should have corresponded to the "Tilting plate" method. In Table 2, in the "Issues" column, the entry "Difficult to place baseline when the RCA is $\sim 90^{\circ}$ " was broken incorrectly in a way that might have suggested that "the RCA is $\sim 90^{\circ}$ " was a separate issue. These errors have been corrected in the HTML and PDF versions of the paper.

Published online: 7 August 2018

https://doi.org/10.1038/s41596-018-0047-0

\section{Publisher Correction: Assessing spatial pattern separation in rodents using the object pattern separation task}

Nick P. van Goethem, Britt T. J. van Hagen and Jos Prickaerts

Correction to: Nature Protocols https://doi.org/10.1038/s41596-018-0013-x, published online 23 July 2018

In the HTML version of this paper originally published online, text in Table 6 was misaligned in a way that made it difficult to determine which entries in the "Problem," "Possible reason," and "Solution" columns corresponded to one another. Additional but less severe alignment problems were also present in the PDF and print articles. These errors have been corrected in the HTML and PDF versions of the paper.

Published online: 10 October 2018

https://doi.org/10.1038/s41596-018-0061-2

\section{Author Correction: Genome-scale CRISPR-Cas9 knockout and transcriptional activation screening}

Julia Joung, Silvana Konermann, Jonathan S Gootenberg, Omar O Abudayyeh, Randall J Platt, Mark D Brigham, Neville E Sanjana and Feng Zhang

Correction to: Nature Protocols 12, 828-863 (2017) https://doi.org/10.1038/nprot.2017.016, published online 23 March 2017

In the published version of this paper, Step 64 of the Procedure reads, "Refer to Steps 37-39 for NGS analysis of the sgRNA distribution." This step should refer the reader to Steps 35-39. This text has not been corrected in the original paper.

Published online: 22 October 2018

https://doi.org/10.1038/s41596-018-0063-0 